\title{
ON THE HAUSDORFF DIMENSION OF JULIA SETS OF SOME REAL POLYNOMIALS
}

\author{
GENADI LEVIN AND MICHEL ZINSMEISTER
}

(Communicated by Mario Bonk)

\begin{abstract}
We show that the supremum for $c$ real of the Hausdorff dimension of the Julia set of the polynomial $z \mapsto z^{d}+c$ ( $d$ is an even natural number) is greater than $2 d /(d+1)$.
\end{abstract}

\section{INTRODUCTION AND STATEMENT OF THE RESULT}

The Julia set of a non-linear polynomial $P: \mathbf{C} \rightarrow \mathbf{C}$ is the set of points having no neighborhood on which the family of iterates $\left(P^{n}\right)$ is normal. It is a compact non-empty set which is, except for very special polynomials $P$, a fractal set. For $c \in \mathbf{C}$ we denote by $f_{c}$ the polynomial

$$
f_{c}(z)=z^{d}+c
$$

where $d \geq 2$ is an even integer number, which we fix. Denote by $J_{c}$ the Julia set of $f_{c}$.

In the quadratic case $d=2$, the values of $c$ for which the Hausdorff dimension (HD) of $J_{c}$ is big has attracted a lot of attention. It has been known since the pioneering work of Douady-Hubbard [3] that the Hausdorff dimension of the Julia set is less than 2 for every hyperbolic polynomial. Thus $H D\left(J_{c}\right)<2$ outside $\partial M$ and the (hypothetic) non-hyperbolic components of (the interior) $M$. We recall that $M$ stands for the Mandelbrot set, that is, the compact subset of $c \in \mathbf{C}$ such that $J_{c}$ is connected. Shishikura 9] was the first to find quadratic Julia sets with Hausdorff dimension 2. He indeed proved that this property holds on a dense $\mathcal{G}_{\delta}$ subset of $\partial M$ or even on a dense $\mathcal{G}_{\delta}$ subset on the boundary of every hyperbolic component of $M$. More recently, Buff and Chéritat 12 have found quadratic Julia sets with positive Lebesgue measure (see also http://annals.math.princeton.edu/articles/3682).

Both Shishikura's and Buff-Chéritat's results are based on the phenomenon of parabolic implosion which was discovered and studied by Douady-Hubbard [3]. It should be pointed out that Buff-Chéritat's result is very involved and that we will make no use of it. There is no doubt that if they exist, values of $c \in \mathbf{R}$ such that the Julia set $J_{c}$ has positive measure must be as hard to find as those of BuffCheritat's. The aim of the present note is much more modest. Its starting point is the second author's re-reading of Shishikura's result [10: it states that if one implodes a polynomial with a parabolic cycle having $q$ petals, then the dimension

Received by the editors November 23, 2010 and, in revised form, October 18, 2011 and January $4,2012$.

2010 Mathematics Subject Classification. Primary 37F10.

The authors were supported in part by the IMPAN-BC European Community Centre of Excellence and by the Marie Curie European network CODY.

(C)2013 American Mathematical Society Reverts to public domain 28 years from publication 
of its Julia set automatically gets bigger than $2 q /(q+1)$. Shishikura's result follows from this by a Baire argument.

Very little is known about Hausdorff dimension of $J_{c}$ for real $c$. In particular, it is not known if, for a given degree $d$,

$$
\sup \left\{H D\left(J_{c}\right), c \in \mathbf{R}\right\}=\mathbf{2} .
$$

Possible candidates for high dimension are of course (just look at them!) infinitely renormalizable polynomials, but the analysis seems to be very delicate, and at least no result concerning dimension 2 has been proven so far (for results in the opposite direction, see [1]). It is unknown for example if the Julia set of the Feigenbaum polynomial has dimension 2 or not (see [4]- 5] for the Julia set of the Feigenbaum universal map). The only known result about this set is a very general result of Zdunik [14: it has dimension bigger than 1 .

If one tries to use the same ideas as in 9 for real polynomials $f_{c}$, one immediately faces the problem that if $f_{c}$, for $c$ real, has a parabolic cycle that may be imploded along the real axis, then the number of petals is 1 and this does not imply more than Zdunik's general result. The only trick of this paper is to make use of a virtual doubling of petals when the critical point is mapped to a parabolic point (by Lavaurs map). It was inspired by the Douady et al. paper [13] and implies the following theorem, which is the main result of this work.

Theorem 1. Let $f_{c}(z)=z^{d}+c$, $d$ even. Let $N$ be the set of parameters $c \in \mathbf{R}$, such that $f_{c}$ has a parabolic cycle of period at least 2 and multiplier 1 . Then there exists an open set $Y$ of $\mathbf{R}$ whose closure contains $N$ such that $J_{c}$ is connected and

$$
H D\left(J_{c}\right)>\frac{2 d}{d+1}
$$

for every $c \in Y$.

Comment 1. In fact, we prove a stronger statement: hyperbolic dimension [9] of $J_{c}$ is bigger than $2 d /(d+1)$.

Comment 2. By [11] (6], 8] for $d=2$ ), the set of real $c$ such that $f_{c}$ is hyperbolic is dense in $\mathbf{R}$. In particular, hyperbolic parameters $c$ are dense in $Y$.

\section{Proof of Theorem 1}

We fix an even integer $d \geq 2$ and consider the family $f_{c}(x)=x^{d}+c$, for real $c$. Then the Julia set $J_{c}$ is connected if and only if $c \in[a, b]$, where $a<0$ is such that $f_{a}^{2}(0)$ is a fixed point and $b>0$ is such that $f_{b}$ has a fixed point with multiplier 1. It is sufficient to prove that, given $c_{0} \in(a, b)$ such that $f_{c_{0}}$ has a neutral cycle of period $k>1$ with multiplier 1 , that is, with one petal, there is an open set $Z$ accumulating at $c_{0}$ for which $H D\left(J_{c}\right)>2 d /(d+1)$ for $c \in \mathbf{Z}$.

We begin with three pictures. The first two illustrate how to choose the parameter (for $d=2$ ) and how the corresponding Julia set looks. The third has kindly been drawn for us by the referee and shows the case $d=4$.

Since $c_{0} \in(a, b)$, the set $J_{c}$ is connected for $c$ in a small neighborhood $U$ of $c_{0}$. It is known that $f_{c}$ has an attracting periodic orbit of period $k$ for $c \in U$ on the left side of $c_{0}$. Since $k>1$, the corresponding filled-in Julia set $K_{c_{0}}$ is such that its interior has a component $\Delta$ containing 0 and has infinitely many preimages of $\Delta$. The boundary $\partial \Delta$ contains a parabolic point $\alpha$ of period $k$. In particular, there is a sequence of preimages of $\Delta$ which intersect the real line and accumulate at $\alpha$. 


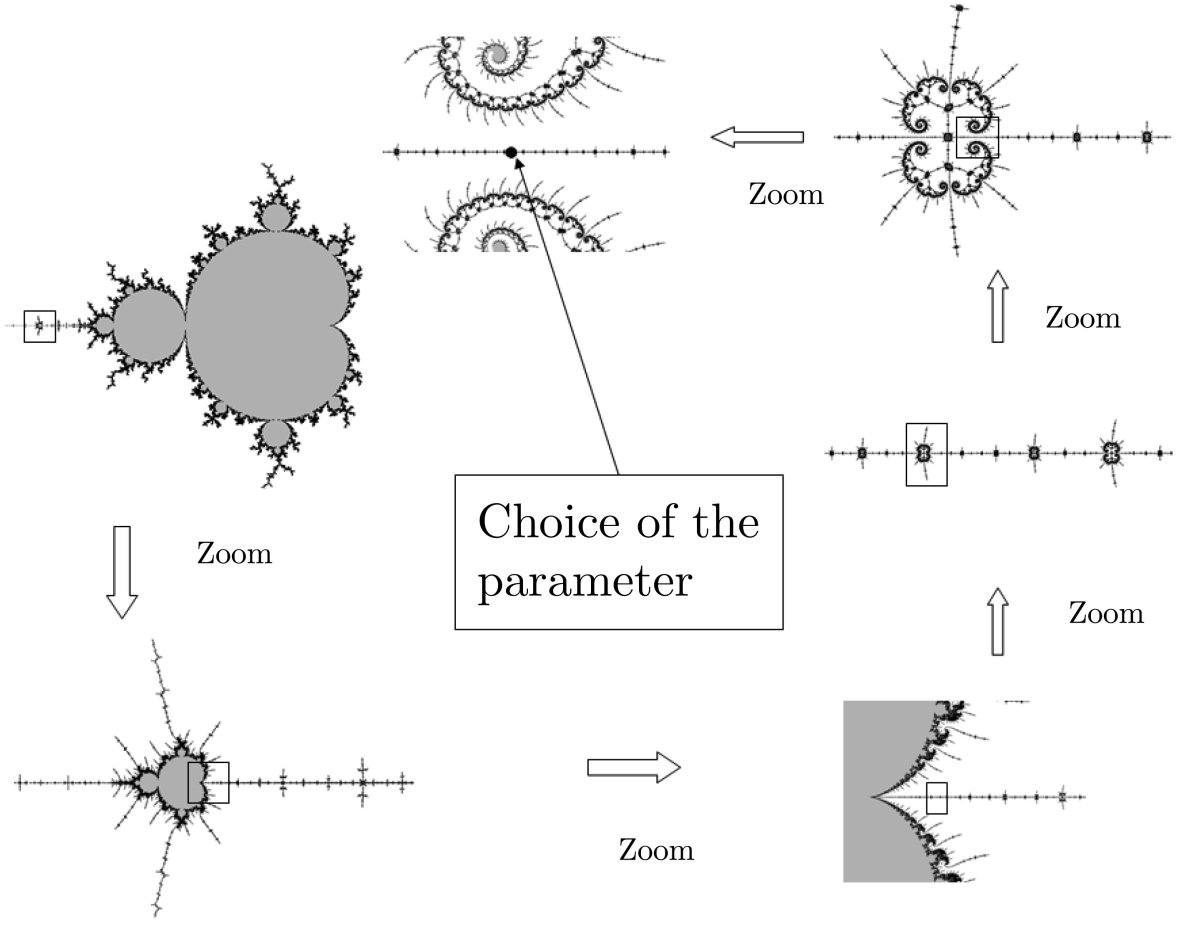

Figure 1. Choice of the parameter
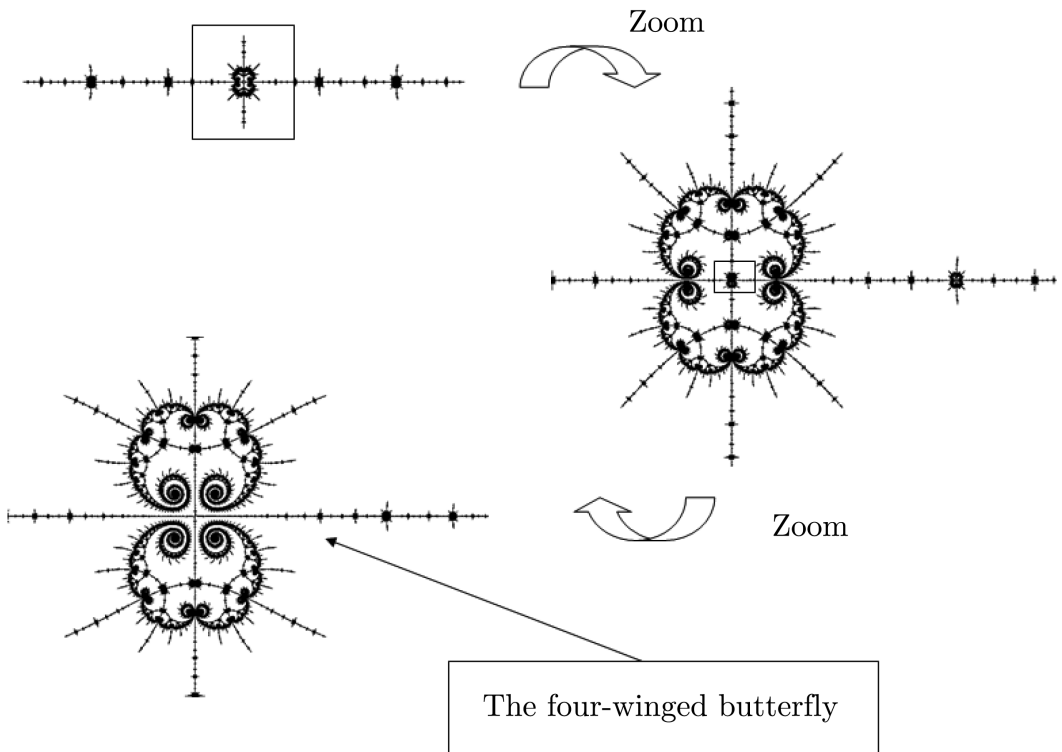

Figure 2. The corresponding Julia set 

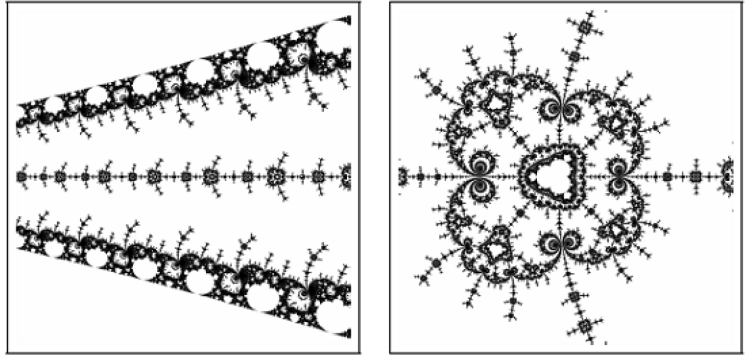

Parameter pictures

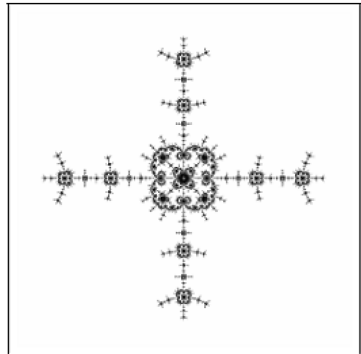

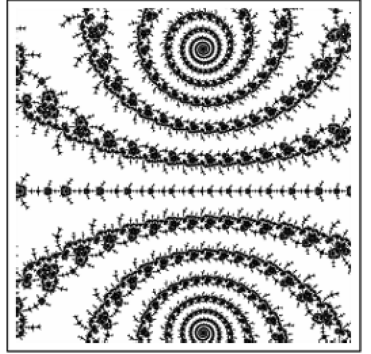

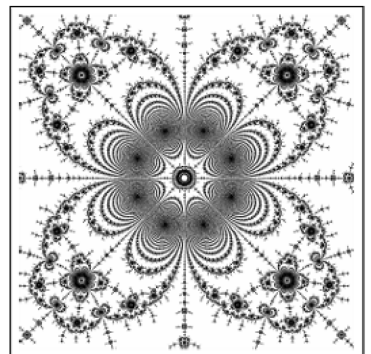

Dynamical pictures

Figure 3. The case $d=4$

For definiteness, one can assume that $\alpha>0$. Then $F=f_{c_{0}}^{k}$ has the following local form:

$$
F(z)=z+a(z-\alpha)^{2}+b(z-\alpha)^{3}+\ldots,
$$

where $a>0$. This implies that a parabolic implosion phenomenon occurs as $\epsilon \rightarrow 0$, $\epsilon>0$, for the maps $f_{c_{0}+\epsilon}$.

At this point we digress somewhat and briefly describe the theory of parabolic implosion.

Let $\delta>0$ be very small and $D_{ \pm}$be the disks centered at $\alpha \pm \delta$ with radius $\delta$. The map $F$ sends $D_{-}$into itself, while $D_{+} \subset F\left(D_{+}\right)$. This defines, after identification of $z$ with $F(z)$ at the boundary, two cylinders $U_{-}=D_{-} \backslash F\left(D_{-}\right)$ and $U_{+}=F\left(D_{+}\right) \backslash D_{+}$. The fact that $U_{ \pm}$are actual cylinders is best seen in the approximate Fatou coordinate $I: z \mapsto-1 /(a(z-\alpha))$ which sends $\alpha$ to $\infty$ and conjugates $F$ to a map which is asymptotically the translation by 1 at $\infty$ :

$$
F_{\infty}(w)=I \circ F \circ I^{-1}(w)=w+1+\frac{A}{w}+O\left(\frac{1}{|w|^{2}}\right),
$$

where $A=1-b / a^{2}$. The real number $A$ is a conformal invariant. In the case of a real polynomial $F$ which has a parabolic fixed point $\alpha$ with multiplier 1 and with a single critical point in its immediate basin $\Delta$, it is known 9 that $A>0$.

By the Riemann mapping theorem these two cylinders may be uniformized by "straight" cylinders. In other words, there exists $\varphi_{ \pm}$mapping the cylinders $U_{ \pm}$to vertical strips $V_{ \pm}$of width 1 conjugating $F$ to the translation by 1 . For further use we notice that, by symmetry, $c_{0}$ being real, we may assume that $\varphi_{ \pm}(\bar{z})=\overline{\varphi_{ \pm}(z)}$. 


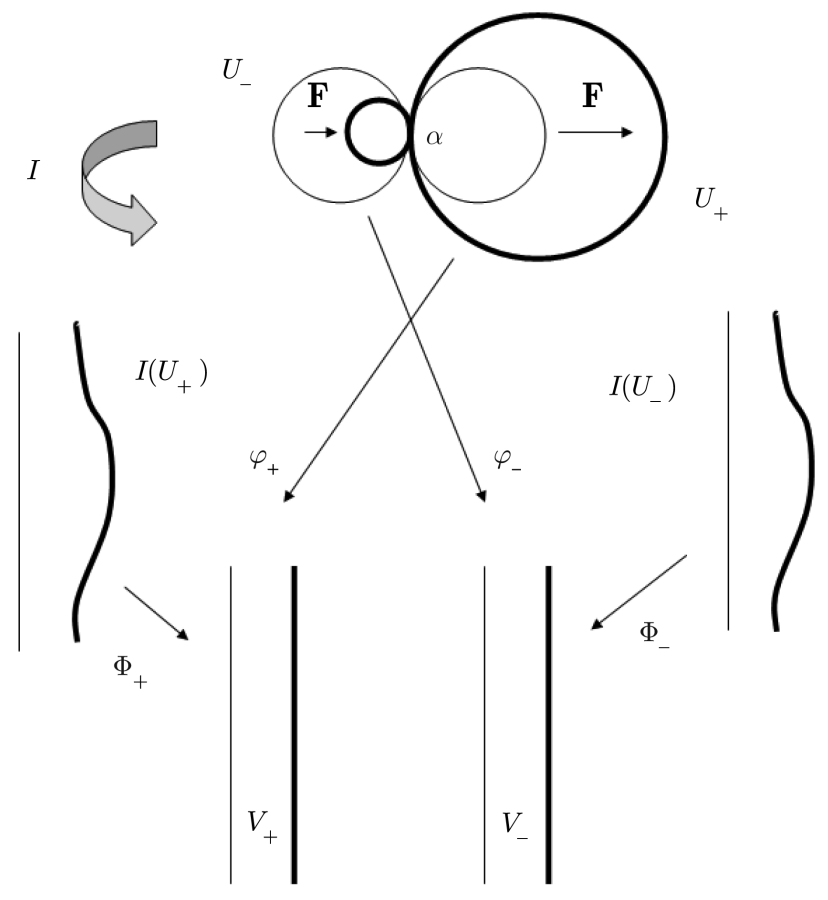

Figure 4. Fatou coordinates

We also notice that these maps are unique up to post-composition by a real translation. These two maps are called respectively repelling $(+)$ and attracting (-) Fatou coordinates. We normalize them as follows. For every $\kappa>0$ and $R>0$, consider two sectors $\Sigma_{-}(\kappa, R)=\{w: \operatorname{Re}(w)>R-\kappa|\operatorname{Im}(w)|\}, \Sigma_{+}(\kappa, R)=\{w$ : $\operatorname{Re}(w)<-R+\kappa|\operatorname{Im}(w)|\}$. Then for any $\kappa>0$ there is a big enough $R(\kappa)$ such that if we introduce two sectors $\Sigma_{ \pm}(\kappa)=\Sigma_{ \pm}(\kappa, R(\kappa))$, then $\varphi_{ \pm}=\Phi_{ \pm} \circ I$, where

$$
\Phi_{ \pm}(w)=w-A \log _{ \pm}(w)+C_{ \pm}+o(1)
$$

as $w \rightarrow \infty$ within $\Sigma_{ \pm}(\kappa)$ respectively. We specify the constants $C_{ \pm}$and the logbranches in such a way that $\Phi_{ \pm}$are real for real $w$. Namely, we choose $C_{-}=0$ and $\log _{-}$to be the standard log-branch in the slit plane $\mathbf{C} \backslash\{\mathbf{x} \leq \mathbf{0}\}$. In turn, let $C_{+}=i A \pi$ and $\log _{+}$be a branch of $\log$ in $\mathbf{C} \backslash\{\mathbf{x} \geq \mathbf{0}\}$ so that $\log _{+}(w)=\log |w|+i \pi$ for $w<0$.

Now, we extend $\varphi_{ \pm}$in the following way. Since

$$
\varphi_{-}(F(z))=T_{1}\left(\varphi_{-}(z)\right),
$$

where $T_{\sigma}$ denotes the translation by $\sigma$, and since every orbit converging to $\alpha$ passes through $U_{-}$exactly once, $\varphi_{-}$extends uniquely to $\Delta$ to a holomorphic function still satisfying (4).

It is seen from (4) that the map $\varphi_{-}: \Delta \rightarrow \mathbf{C}$ is a branched covering, with the critical points at 0 and all its preimages in $\Delta$ by $F^{n}, n>0$, and the critical values at the real numbers $\varphi_{-}(0)-n, n \geq 0$. In particular, there exists a simply-connected domain $\Omega_{-} \subset \Delta \cap \mathbf{H}^{+}$, where $\mathbf{H}^{+}$is the upper half-plane, such that $\varphi_{-}: \Omega_{-} \rightarrow \mathbf{H}^{+}$ 
is a holomorphic homeomorphism. Moreover, the intersection of the boundary of $\Omega_{-}$with $\mathbf{R}$ is the interval $(0, \alpha)$.

Concerning the repelling Fatou coordinate it is best to consider $\psi_{+}=\varphi_{-}^{-1}$ : $V_{+} \rightarrow U_{+}$. The functionnal relation is now

$$
\psi_{+}\left(T_{1}(z)\right)=F\left(\psi_{+}(z)\right),
$$

and we can extend $\psi_{+}$to an entire function by putting, for $n \in \mathbf{Z}, \psi_{+}\left(T_{n}(z)\right)=$ $F^{n}\left(\psi_{+}(z)\right)$ for $z \in V_{+}$. There exists a simply-connected domain $\Omega_{+} \subset \mathbf{H}^{+}$such that $\psi_{+}: \Omega_{+} \rightarrow \mathbf{H}^{+}$is a homeomorphism.

Now let $\sigma$ be a real number. We define the Lavaurs map $g_{\sigma}$ on the component $\Delta$ of the interior of the filled-in Julia set of $f_{c_{0}}$ by

$$
g_{\sigma}=\psi_{+} \circ T_{\sigma} \circ \varphi_{-} .
$$

The raison d'être of this definition is the following theorem due to Douady and Lavaurs [2]:

Theorem 2. There exists a sequence of positive $\epsilon_{n}$ converging to 0 and a sequence of positive integers $N_{n}$ such that

$$
g_{\sigma}(z)=\lim _{n \rightarrow \infty} f_{c_{0}+\epsilon_{n}}^{k N_{n}}(z)
$$

uniformly on compact sets of $\Delta$.

Using (3) with the constants $C_{ \pm}$and the log-branches specified above, it is easy to get that, for every $\kappa$, if $w$ tends to $\infty$ in $\Sigma(\kappa):=\Sigma_{-}(\kappa) \cap \Sigma_{+}(\kappa) \cap \mathbf{H}^{+}$, then $g_{\infty}(w):=I \circ g_{\sigma} \circ I^{-1}(w)=w+(\sigma-i A \pi)+O\left(\frac{1}{|w|}\right)$, where $A$ is real and positive. Therefore, for every real $\sigma$ and every $\kappa>\kappa(\sigma)$, the inverse map $g_{\infty}^{-1}$ leaves the sector $\Sigma(\kappa)$ invariant and $g_{\infty}^{-n} \rightarrow \infty$ as $n \rightarrow \infty$. Coming back to the $z$-plane, we conclude that the branch $G=I^{-1} \circ g_{\infty}^{-1} \circ I$ of $g_{\sigma}^{-1}$ leaves the set $S(\kappa)=I^{-1}(\Sigma(\kappa))$ invariant and $G^{n}(z) \rightarrow \alpha$ as $n \rightarrow \infty$, for $z \in S(\kappa)$ and every $\kappa>\kappa(\sigma)$. We have, for $w \in \Sigma(\kappa)$,

$$
G_{\infty}(w):=I \circ G \circ I^{-1}(w)=w+(-\sigma+i A \pi)+O\left(\frac{1}{|w|}\right) .
$$

Now, from the definition of $g_{\sigma}(z)$ and the global properties of the maps $\varphi_{-}$and $\psi_{+}$, there follows the existence of a simply-connected domain $\Omega_{0} \subset \Omega_{-}$, which is mapped by $g_{\sigma}$ homeomorphically onto $\mathbf{H}^{+}$and such that $\alpha \in \bar{\Omega}_{0}$. Moreover, from the above description, $S(\kappa) \subset \Omega_{0}$, for every $\kappa>\kappa(\sigma)$. Therefore, the branch $G$ of $g^{-1}$ which is defined above extends to a global univalent branch $G: \mathbf{H}^{+} \rightarrow \Omega_{0}$ of $g_{\sigma}^{-1}$. Since $\Omega_{0} \subset \mathbf{H}^{+}$, the iterates $G^{n}: \mathbf{H}^{+} \rightarrow \Omega_{0}, n>0$, converge uniformly on compact sets in $\mathbf{H}^{+}$to a unique fixed point in $\bar{\Omega}_{0}$, which must be $\alpha$.

Let us consider the continuous map $\sigma \mapsto g_{\sigma}(0)=\psi_{+}\left(\varphi_{-}(0)+\sigma\right)$ : if $\sigma$ runs in the interval $I=\left\{\varphi_{+}(x)-\varphi_{-}(0): x \in U_{+} \cap \mathbf{R}\right\}$, then $g_{\sigma}(0)$ runs over $U_{+} \cap \mathbf{R}$. It is thus clear, and this is the key point in the proof, that we can choose $\sigma$ in such a way that $g_{\sigma}(0)$ is a preimage of $\alpha$ : there is $j \geq 1$ such that $f_{c_{0}}^{j} \circ g_{\sigma}(0)=\alpha$. Since 0 is a critical point for $g_{\sigma}$, taking the inverse image by $f_{c_{0}}^{j} \circ g_{\sigma}$ has the same effect as multiplying the number of petals by $d$, and we may state:

Lemma 2.1. There exists an infinite iterated function system defined on a small compact neighborhood $B_{0}$ of zero and generated by some holomorphic branches of $f_{c_{0}}^{-1}$ and $g_{\sigma}^{-1}$ such that its limit set has Hausdorff dimension bigger than $2 d /(d+1)$. 
Comment 3. In fact, the limit set is a subset of a so-called Julia-Lavaurs set denoted by $J_{c_{0}, \sigma}$. It is defined as follows. The map $g_{\sigma}$ can be extended in a natural way from $\Delta$ to the interior of the filled-in Fatou set of $f_{c_{0}}$ : if $f_{c_{0}}^{k}(z) \in \Delta$, set $g_{\sigma}(z)=g_{\sigma} \circ f_{c_{0}}^{k}(z)$. Then $J_{c_{0}, \sigma}$ is simply the closure of the set of points $z$ for which there exists $m \in \mathbf{N}$ such that $g_{\sigma}^{m}(z)$ is defined and belongs to $J\left(f_{0}\right)$.

This lemma together with the above discussion implies the theorem. Indeed, by a general property of iterated function systems [7, there exists its finite subsystem with the Hausdorff dimension of its limit set bigger than $2 d /(d+1)$. On the other hand, the finite iterated function system persists for $f_{c_{0}+\epsilon}$ by (6). To be more precise, if $\left\{I_{j}: B_{0} \rightarrow X_{j}, 1 \leq j \leq j_{0}\right\}$ is this finite iterated function system, then each $I_{j}$ can be extended to a univalent map to a fixed neighborhood $Y$ of $B_{0}$ as $I_{j}: Y \rightarrow Y_{j}$. Consider the inverse univalent map $I_{j}^{-1}: Y_{j} \rightarrow Y$. Since the convergence in (6) is uniform on compacts in $\Delta$, for every $\epsilon_{n}$ small enough there is some integer $N_{j}>0$ and a compact set $X_{j, n}$ so that $f_{c_{0}+\epsilon_{n}}^{N_{j}}: X_{j, n} \rightarrow B_{0}$ is univalent too. Now it is clear that, for every $\epsilon_{n}$ small enough, the non-escaping set $K_{n}$ of the dynamical system which consists of finitely many maps $f_{c_{0}+\epsilon_{n}}^{N_{j}}: X_{j, n} \rightarrow B_{0}$, $1 \leq j \leq j_{0}$, has the Hausdorff dimension which is bigger than $2 d /(d+1)$. On the other hand, $K_{n}$ must lie in the Julia set of $f_{c_{0}+\epsilon_{n}}$ because some iterate of the map $f_{c_{0}+\epsilon_{n}}^{N_{1} N_{2} \ldots N_{j_{0}}}$ leaves the set $K_{n}$ invariant and is expanding on it.

Proof of Lemma 2.1. As the first step, let us fix a small enough closed ball $B_{0}$ around zero so that it does not contain points of the postcritical set of $f_{c_{0}}$. There exists its preimage $B^{\prime}$ by $F^{-1}$ in $\Delta \cap \mathbf{H}^{+}$. Then we can apply to $B^{\prime}$ the maps $G^{n}$, $n>0$. By the above, $B_{n}^{\prime}=G^{n}\left(B^{\prime}\right)$ are pairwise disjoint, compactly contained in $\Delta$, and $B_{n}^{\prime} \rightarrow \alpha$ as $n \rightarrow \infty$. Now, for every $n \geq n_{0}$, so that $B_{n}^{\prime}$ lies in a small enough neighborhood $U$ of $\alpha$, we make "clones" of $B_{n}^{\prime}$ in $U \cap \Delta$, applying to it $F^{r}, r \in \mathbf{Z}$, where $F^{r}$ for $r<0$ is a well-defined in $U \cap \Delta$ branch which fixes $\alpha$. We obtain the sets $B_{n, r}^{\prime}=F^{r}\left(B_{n}^{\prime}\right)$. In the second step, we consider the map $f_{c_{0}}^{j} \circ g_{\sigma}$ from a neighborhood $V$ of 0 onto $U$. This map is a ramified cover with the only ramification point at 0 of order $d$. Let $U^{*}=U \backslash\{x \geq \alpha\}$ and $V^{*}=V \cap\{z: \operatorname{Arg}(z) \in(0,2 \pi / d)\}$. Denote by $h$ a branch of $\left(f_{c_{0}}^{j} \circ g_{\sigma}\right)^{-1}$ from $U^{*}$ onto $V^{*}$. Let $B_{n, r}=h\left(B_{n, r}^{\prime}\right)$. We obtain a system of holomorphic maps $\boldsymbol{\Psi}=\left\{\psi_{n, r}: B_{0} \rightarrow B_{n, r}\right\}$, where $\psi_{n, r}=$ $h \circ F^{r} \circ G^{n} \circ F^{-1}, r \in \mathbf{Z}, n \geq n_{0}$. If the neighborhood $U$ is chosen small enough, the maps $\psi_{n, r}$ extend to univalent maps in a fixed neighborhood $\tilde{B}$ of $B_{0}$ into itself. In particular, the compact sets $B_{n, r}$ are pairwise disjoint and compactly contained in $B_{0}$. Now, it is quite standard to check that $\boldsymbol{\Psi}$ forms a conformal infinite iterated function system in the sense of [7] (strictly speaking, in the hyperbolic metric of $\tilde{B}$, which is equivalent to the Euclidean one on $B_{0}$ ). Let us calculate the parameter $\theta=\inf \{t: \psi(t)<\infty\}$ of $\boldsymbol{\Psi}$, where $\psi(t)=\sum_{(n, r)} \max _{z \in B_{0}}\left|\psi_{n, r}^{\prime}(z)\right|^{t}$. The map $\psi_{n, r}=h \circ I^{-1} \circ F_{\infty}^{r} \circ G_{\infty}^{n} \circ I \circ F^{-1}$. Here $F^{-1}$ is a univalent map of a neighborhood $\tilde{B}$ of $B_{0}$ into $\Delta$. Now, routine and well-known calculations based on (2)-(7) show (see e.g. [10]) that, for all $r \in \mathbf{Z}, n \geq n_{0}$ and some $C$, which depends only on a compact set in $\mathbf{H}^{+} \cap \Delta$, from which $w$ is taken, $C^{-1}|r+(-\sigma+i \pi A) n| \leq\left|F_{\infty}^{r} \circ G_{\infty}^{n}(w)\right| \leq$ $C|r+(-\sigma+i \pi A) n|$ and $C^{-1} \leq\left|\left(F_{\infty}^{r} \circ G_{\infty}^{n}\right)^{\prime}(w)\right| \leq C$. On the other hand, the map $h$ is a composition of a univalent map with an inverse branch of $z^{1 / d}$. This gives us $C_{1}^{-1}|r+(-\sigma+i \pi A) n|^{-1-1 / d} \leq\left|\psi_{n, r}^{\prime}(z)\right| \leq C_{1}|r+(-\sigma+i \pi A) n|^{-1-1 / d}$, for some 
$C_{1}$ and every $z \in B_{0}$. It follows that the series for $\psi(t)$ converges if and only if $t>\theta=2 d /(d+1)$ and $\psi(\theta)=\infty$. Hence [7], the Hausdorff dimension of the limit set of $\boldsymbol{\Psi}$ is strictly bigger than $2 d /(d+1)$.

\section{ACKNOWLEDGMENT}

This work was done during the first author's one-month visit at the University of Orléans in 2008.

\section{REFERENCES}

1. A. Avila and M. Lyubich, Examples of Feigenbaum Julia sets with small Hausdorff dimension, Dynamics on the Riemann Sphere, in honor of B. Branner 1 (2006), 71-87. MR.2348955 (2008j:37102)

2. A. Douady, Does a Julia set depend continuously on the polynomial?, Proceedings of Symposia in Applied Mathematics 49 (1994), 91-135. MR.1315535

3. A. Douady and J. Hubbard, Etude dynamique des Polynômes complexes: Première partie, Publications Mathématiques d'Orsay 84-02 (1984), 1-75. MR762431 (87f:58072a)

4. G. Levin and G. Swiatek, Hausdorff dimension of Julia sets of Feigenbaum type, Communications in Mathematical Physics 258 (2005), 135-148. MR2166843 (2007b:37104)

5. Measure of the Julia set of the Feigenbaum map with high criticality, Ergodic Theory and Dynamical Systems 30 (2009), 855-875. MR2643714 (2011f:37066)

6. J. Graczyk and G. Swiatek, Generic hyperbolicity in the logistic family, Annals of Mathematics 146 (1997), 1-52. MR1469316 (99b:58079)

7. R. D. Mauldin and M. Urbanski, Dimension and measure in infinite iterated function systems, Proc. London Math. Soc. 73 (1996), 105-154. MR.1387085 (97c:28020)

8. M. Lyubich, Dynamics of quadratic polynomials, Acta Mathematica 178 (2007), 185-297. MR.1459261 (98e:58145)

9. M. Shishikura, The Hausdorff dimension of the boundary of the Mandelbrot set and Julia sets, Annals of Mathematics 147 (1998), 225-267. MR1626737 (2000f:37056)

10. M. Zinsmeister, Fleur de Leau-Fatou et dimension de Hausdorff, Comptes-rendus de l'Académie des Sciences 326 (1998), 1227-1232. MR.1650223 (99j:58180)

11. O. Kozlovsky, W. Shen, and S. van Strien, Density of hyperbolicity in dimension one, Annals of Mathematics 166 (2007), 145-182. MR2342693 (2008j:37081)

12. X. Buff and A. Chéritat, Ensembles de Julia quadratiques de mesure strictement positive, Comptes-rendus de l'Académie des Sciences 341 (2005), 669-674. MR2183346 (2006k:37133)

13. X. Buff, R. Devaney, A. Douady, and P. Sentenac, Baby Mandelbrot sets are born in cauliflowers, London Mathematical Society Lecture Notes Series 274 (2000), 19-36. MR.1765083 (2001g:37062)

14. Anna Zdunik, Parabolic orbifolds and the dimension of the maximal measure for rational maps, Inventiones Mathematicae 99 (1990), 627-649. MR 1032883 (90m:58120)

Institute of Mathematics, Hebrew University of Jerusalem, Jerusalem 91904, Israel MAPMO, Université D'Orléans, BP 6759, 45067 Orléans Cedex 2, France 